\title{
Oliver C. Wells' Vision: Use of Low-Loss Electrons to Enhance and Measure the Surface Detail in the Scanning Electron Microscope at High Resolution
}

\author{
Michael T. Postek, Andras E. Vladar, and Bin Ming ${ }^{1}$ \\ ${ }^{1}$ National Institute of Standards and Technology, Physical Measurement Laboratory, Gaithersburg, MD \\ $20899^{[1]}$
}

Low-loss electron (LLE) imaging in the scanning electron microscope (SEM) is based on the collection of backscattered electrons that have undergone minimal elastic interactions within a sample and therefore carry high-resolution, surface-specific information. Oliver Wells credits the concept of filtering the high-energy emitted electrons in the SEM to a statement made by McMullan [2], that the signal collected in the SEM could be "...restricted to the electrons which have lost only small amounts of energy and which have therefore travelled only short distances through the specimen." But, it was Oliver Wells who carried this concept forward to become the viable LLE imaging technique [3, 4]. He developed the theory, designed and assembled a working LLE detector capable of demonstrating that concept. For many years, he researched and refined the LLE technique to fulfill his vision of collecting the highest resolution surface information possible from the SEM.

Oliver Wells described extensively the LLE technique in a number of papers [such as reference 5]. During this work, Wells not only imaged successfully conducting, but also non-conducting samples such as uncoated photoresist at high electron landing energies (accelerating voltages) [6]. This early work did not extend this technique to low electron landing energy applications, such as those then commonly employed in semiconductor metrology for process control where the typical secondary electron (SE) image was used. Alternatively, the conventional backscattered electron (BSE) signal was shown to be useful at low landing energies using microchannel-plate electron detectors for both SE and BSE collection [7]. It was anticipated that application of the LLE method could potentially provide similar results but with increased sharpness. Unfortunately, since fewer BSE are generated than SE, without using frame storage methods or field emission electron sources the signal-to-noise ratio $(\mathrm{S} / \mathrm{N})$ in both cases is very poor. However, optimizing operating conditions in experiments comparing similar SE vs. BSE imaging showed that similar measurements done with backscattered electrons report typically smaller (closer to reality) dimensions of the same structures than those with the SE image [7]. This difference was documented for semiconductor production and can be as great as $10 \%$. Additionally, collection and measurement of the BSE signal demonstrated higher precision. This mode of electron collection was adopted for semiconductor production applications by one critical dimension (CD) scanning electron microscope manufacturer, until throughput issues became critical.

In order to investigate the potentials of the application of LLE imaging to semiconductor metrology, collaborative work at the National Institute of Standards and Technology (NIST) was initiated with Oliver Wells to explore LLE for accurate dimensional standards development and semiconductor process control [8]. The work undertaken at NIST sought to reduce some of the geometrical limitations by using an instrument with the (then) new in-lens detector design. It also sought to improve the $\mathrm{S} / \mathrm{N}$ problems through the use of frame storage and a high-brightness field-emission electron gun, and assessing the capabilities for LLE imaging for accurate metrology at low accelerating voltages. 
Overall, this collaborative work demonstrated that, given the proper geometries, the LLE signal does provide more surface specific information, especially at low landing energies than the SE image (Fig. 1) and that additionally the LLE image is more readily modeled. LLE detection is possibly the best signal for accurate SEM-based dimensional metrology and 3-D surface imaging. However, as presently implemented, the $\mathrm{S} / \mathrm{N}$ and geometric considerations remain less favorable than with the standard $\mathrm{SE}$ imaging method, thus compromising throughput. Therefore, it was concluded that if accurate SEM metrology is desired using this methodology, a properly designed, dedicated CD Metrology tool based upon LLE instrumentation would be necessary. The value of LLE has not been fully exploited, but has not been forgotten. Some of these early results and further experimental and modeling work that continued the exploration into the possibilities that LLE affords to accurate metrology and to determine the necessary information related to geometric and design parameters necessary for implementation, will be discussed.

\section{References:}

[1] Contribution of the National Institute of Standards and Technology; not subject to copyright.

[2] D. McMullan, Proc. Inst. Elec. Eng (London), Pt. B 100, (1953), p. 245-256.

[3] O. C. Wells, Appl Phys Lett 16(4), (1970), p.151-152.

[4] O. C. Wells, Appl Phys Lett 19(7), (1971), p. 232-235.

[5] O. C. Wells, Scan Electron Microsc, 1, IITRI Chicago, (1972), p. 43-50.

[6] O. C. Wells, Appl. Phys. Lett. 49(13), (1986) p. 764-766.

[7] M. Postek et al., SCANNING 23(5), (2001), p. 298-304.

[8] M. Postek et al., Rev Sci. Instrum, 61(12), (1990), p. 3750-3754.

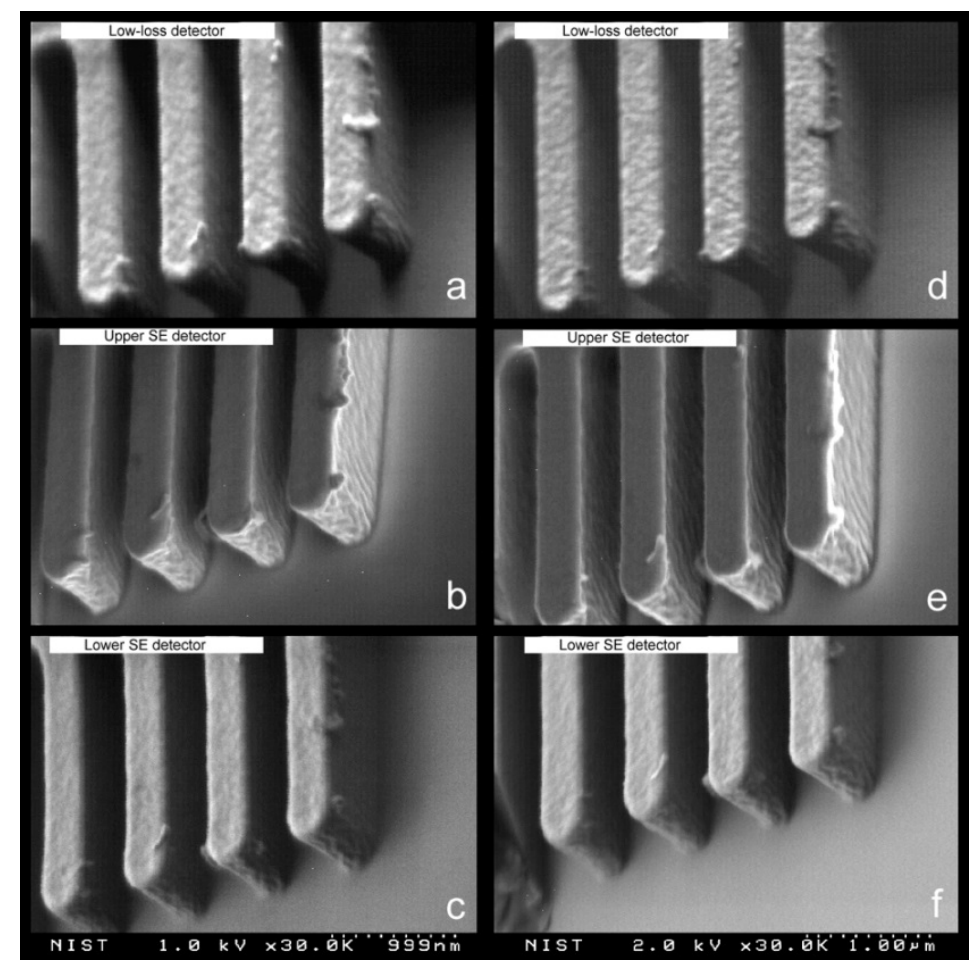

Figure 1. Comparative images of a photoresist sample taken at $2 \mathrm{kV}(1-\mathrm{c})$ and $1 \mathrm{kV}(\mathrm{d}-\mathrm{f})$; the micrographs are marked with the detector type used. The field width of each image is $3.5 \mu \mathrm{m}$ (from reference 7). 